\title{
CLASSIC DANDY WALKER MALFORMATION: ANTENATAL SONOGRAPHIC FINDINGS AND POSTNATAL STATUS
}

Arvinder Singh ${ }^{1}$, Poonam Ohri², Sohan Singh 3 , Manjeet Kaur ${ }^{4}$

\section{HOW TO CITE THIS ARTICLE:}

Arvinder Singh, Poonam Ohri, Sohan Singh, Manjeet Kaur. "Classic Dandy Walker Malformation: Antenatal Sonographic Findings and Postnatal Status". Journal of Evolution of Medical and Dental Sciences 2014; Vol. 3, Issue 23, June 09; Page: 6407-6412, DOI: $10.14260 /$ jemds/2014/2756

ABSTRACT: Dandy Walker malformation is a rare congenital abnormality that affects the cerebellum and some of its components; particularly hypoplasia of cerebellar vermis, a cystic dilatation of fourth ventricle and is characterized by an enlarged posterior fossa. Here we present a case of classical DWM with antenatal and postnatal imaging.

KEYWORDS: Dandy walker malformation, antental, postnatal ,imaging.

INTRODUCTION: Dandy-Walker malformation is a rare congenital abnormality that affects the cerebellum and some of its components; particularly hypoplasia of cerebellar vermis, a cystic dilatation of fourth ventricle and is characterized by an enlarged posterior fossa.[1] Dandy-Walker malformation was originally described in 1887 by Sutton and further characterized by Dandy and Black fan in 1914 followed by Tagart and Walker in 1942. Benda finally labeled this disease as dandy walker in 1954.[2]

Dandy-Walker malformation has a reported incidence of 1 in 25,000-35,000 live births with a slight female predominance. It accounts for $1-4 \%$ of cases of antenatally detected hydrocephalus.[3]

Cystic or cyst-like malformations of the posterior fossa represent a spectrum of disorders, including the Dandy-Walker malformation, vermian-cerebellar hypoplasia, mega cisterna magna, and arachnoid cyst. The classic Dandy-Walker malformation comprises of the triad of hypoplasia of the vermis and cephalad rotation of the vermian remnant; cystic dilatation of the fourth ventricle extending posteriorly; an enlarged posterior fossa with torcular-lambdoid inversion (torcular lying above the level of the lambdoid due to abnormally high tentorium.It is at the severe end of the spectrum denoted by the term Dandy-Walker continuum. ${ }^{[4]}$

Genetic factors plays a major role in the etiology of DWM, may occur as part of Mendelian disorders and chromosomal aberrations. Environmental factors including viral infections, alcohol and diabetes have also been suggested in the genesis of DWM but the evidence is uncertain.[13]

CASE REPORT: A 25 years old lady $\mathrm{G}_{2} \mathrm{P}_{1} \mathrm{~L}_{1}$ presented to the antenatal OPD at 24 weeks of gestation. She was married for the last 4years with no h/o consanguinity. Her first pregnancy and delivery were uneventful and she had a 2years old daughter. Her routine antenatal scan at 24 weeks showed a single viable pregnancy with a large posterior fossa cyst communicating with the fourth ventricle through a large gap due to absence of cerebellar vermis giving key hole appearance [Fig. 1,2].

The diagnosis of classical DWM was made antenatally. The patient opted for continuation of the pregnancy.

After about 3-4 months of post natal period, the child developed features of hydrocephalus. Non contrast CT scan was done which showed marked degree of hydrocephalus with thinning of the cerebral cortex. A large posterior fossa cyst was detected as in antenatal sonography which was 
communicating with the dilated fourth ventricle. Complete absence of cerebellar vermis was seen. The cerebellar hemispheres were splayed and displaced anterolaterally and compressed against the posterior walls of the petrous temporal bones [Fig. 3a, b].

Later intracranial shunting was done to relieve the raised intracranial tension due to marked hydrocephalus [Fig.3c, d].However, in course of time the child expired with various complications.

DISCUSSION: The Dandy-Walker complex is a rare congenital intracranial malformation that comprises a spectrum of posterior fossa abnormalities. Dandy-Walker malformation consists of cystic dilatation of the 4th ventricle, complete or partial agenesis of the cerebellar vermis and an enlarged posterior fossa, Dandy-Walker variant consists of cystic posterior fossa mass with variable inferior hypoplasia of the cerebellar vermis with no enlargement of the posterior fossa and Megacisterna magna is enlarged cisterna magna with normal cerebellar vermis and fourth ventricle. ${ }^{[9,11]}$

DWM is associated with other intracranial anomalies such as agenesis of the corpus callosum, holoprosencephaly, occipital encephaloceles and ocular abnormalities. Extracranial anomalies include polycystic kidneys, cardiovascular defects, polydactyly and cleft palate. Postnatal studies indicate that the incidence of associated malformations range between 50 and 70\%.[9]

The sonographic features of DWM are partial or complete absence of cerebellar vermis with a large posterior fossa cyst. Dandy walker variant is defined as only partial (inferior) absence of cerebellar vermis. Other central nervous system findings includes hydrocephalus and agenesis of the corpus callosum. ${ }^{[5,6,11]}$

Prenatal diagnosis of Dandy-Walker malformation should not be made before the 18th week of gestation because development of the cerebellar vermis may be incomplete at that time. Intracranial findings in fetuses may vary, however sagittal measurements exceeding $10 \mathrm{~mm}$ help confirm the presence of a Dandy-Walker cyst. [7]

NCCT scan delineates various components of Dandy-Walker malformation, which includes partial or complete absence of the cerebellar vermis, posterior fossa cyst contiguous with the fourth ventricle, small and widely separated cerebellar hemispheres, anterior and lateral displacement of hypoplastic cerebellar hemispheres and hydrocephalus. CT is used to diagnose Dandy-Walker malformation and to follow ventricular shunt function in shunted patients. ${ }^{[8]}$

A correct diagnosis cannot be made without a good quality MRI including sagittal views of the vermis and T2-weighted images. The diagnosis of DWM to those malformations with all of the following features: a large median posterior fossa cyst widely communicating with the fourth ventricle, a small, rotated, raised cerebellar vermis, an upwardly displaced tentorium, an enlarged posterior fossa, antero-laterally displaced but apparently normal cerebellar hemispheres, a normal brain stem. If any one of the previous criteria were not met, the malformation was considered distinct from DWM. ${ }^{[9,10]}$

In $80 \%$ of the cases, post-natal complication of hydrocephalus often develop within 3 months after birth.[10] Cystperitoneal shunting avoids the risk of an entrapped fourth ventricle and is presently the best surgical procedure. The overall mortality is about $12.5 \% .{ }^{[11,12]}$

CONCLUSION: DWM is a condition that can be diagnosed by advanced imaging modalities which are expensive to use in developing countries. Various imaging modalities like antenatal sonography can be useful to diagnose the disease at the earliest. MRI better delineates associated abnormalities and CT scan helps in localization and patency of the intracranial shunt. 


\section{REFERENCES:}

1. Ecker JL, Shipp TD, Bromley B, Benacerraf B. The sonographic diagnosis of dandy-walker and dandy-walker variant: Associated findings and outcomes. Prenat Diagn 2000; 20: 328-32.

2. Cardoso J, Lange MC, Lorenzoni PJ, Scola RH, Werneck LC. Dandy-walker syndrome in adult mimicking myasthenia gravis. Arq Neuropsiquiatr 2007; 65: 173-5.

3. T Lavanya, M Cohen, S V Gandhi, T Farrell, E H Whitby. A case of a Dandy-Walker variant: the importance of a multidisciplinary team approach using complementary techniques to obtain accurate diagnostic information. BJR 2008; 81: 970, e242-e245.

4. SGM Caroll, H Porter, SA Fatah, PM Kyle, PW Soothill. Correlation of the prenatal ultrasound diagnosis and pathological findings in fetal brain abnormalities. Ultrasound Obstet Gynaecol 2000; $16 ; 149-153$.

5. O Klien, A Pierre Kahn, N boddaert, D Parisot, F Brunelle. Dandy-Walker malformation: prenatal diagnosis and prognosis. Child's nervous system. 2003; 19(7-8): 484-489.

6. A Alam, BN Chander, M Bhatia. Dandy-Walker Variant: Prenatal Diagnosis by Ultrasonography. MJAFI 2004; 60: 287-289.

7. DA Nyberg, DR Cyr, LA Mack, J Fitzsimmons, D Hickok, BS Mahony. The Dandy-Walker malformation prenatal sonographic diagnosis and its clinical significance. Journal of ultrasound in medicine 1988; 7 (2): 65-71.

8. Lutfi Incesu et al. Imaging in Dandy-Walker Malformation. Online-updated may 2013. http://emedicine.medscape.com/article/408059-overview.

9. Kollias SS, Ball WS, Prenger EC. Cystic malformations of the posterior fossa: differential diagnosis clarified through embryologic analysis. Radiographics. 1993; 13 (6): 1211-31.

10. Pietro Spennato, Giuseppe Mirone, Anna Nastro, Maria Consiglio Buonocore, Claudio Ruggiero, Vincenzo Trischitta, Ferdinando Aliberti, Giuseppe Cinalli. Hydrocephalus in Dandy-Walker malformation. Childs Nerv Syst 2011; 27: 1665-1681.

11. Sreelatha S, Vedavathy Nayak, Sathya P, Nirmala Hanji. Dandy-Walker Variant: A Case Report. Sch J Med Case Rep 2014; 2(1): 40-41.

12. Jean Francios Hirsch, Alain Pierre Kahn, Domnique Renier, Christian Sainte Rose, Elisabeth Hoppe Hirsch. Dandy walker malformation: a review of 40 cases. Journal of Neurosurgery 1984; 61(3): 515-522.

13. Jyothi Tadakamadla, Santhosh Kumar1, G. P. Mamatha. Dandy-Walker malformation: An incidental finding. Indian Journal of Human Genetics 2010; 16(1): 33-35. 


\section{CASE REPORT}

Fig. 1: (a, b)-Antenatal Sonogram of Fetal Brain in axial planes showing features of classical Dandy-Walker malformation; a posterior fossa cyst communicating with the dilated fourth ventricle due to absence of cerebellar vermis and splaying of the cerebellar hemispheres (arrows). Mild dilatation of lateral ventricles was also noted.

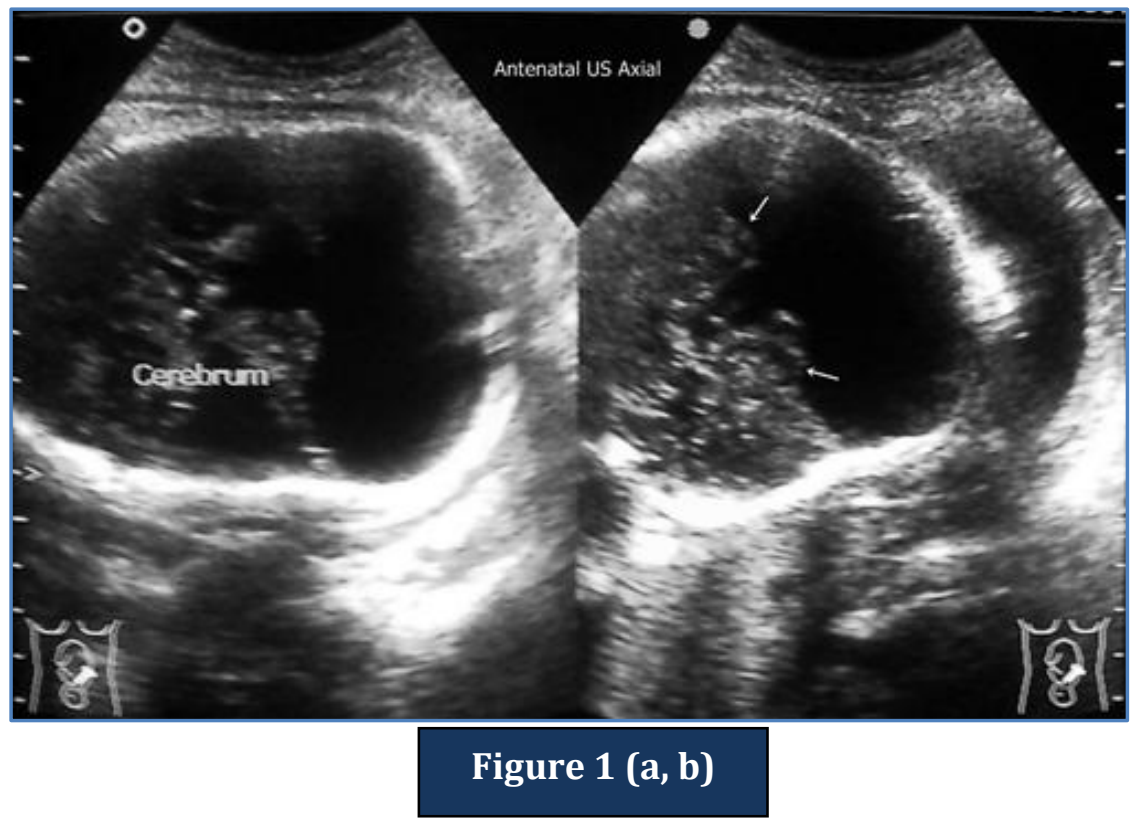

Fig. 2: Antenatal Sonogram of Fetal Brain in sagittal plane showing features of classical Dandy-Walker malformation; a posterior fossa cyst communicating with the dilated fourth ventricle due to absence of cerebellar vermis and splaying of the cerebellar hemispheres; raised tentorium.

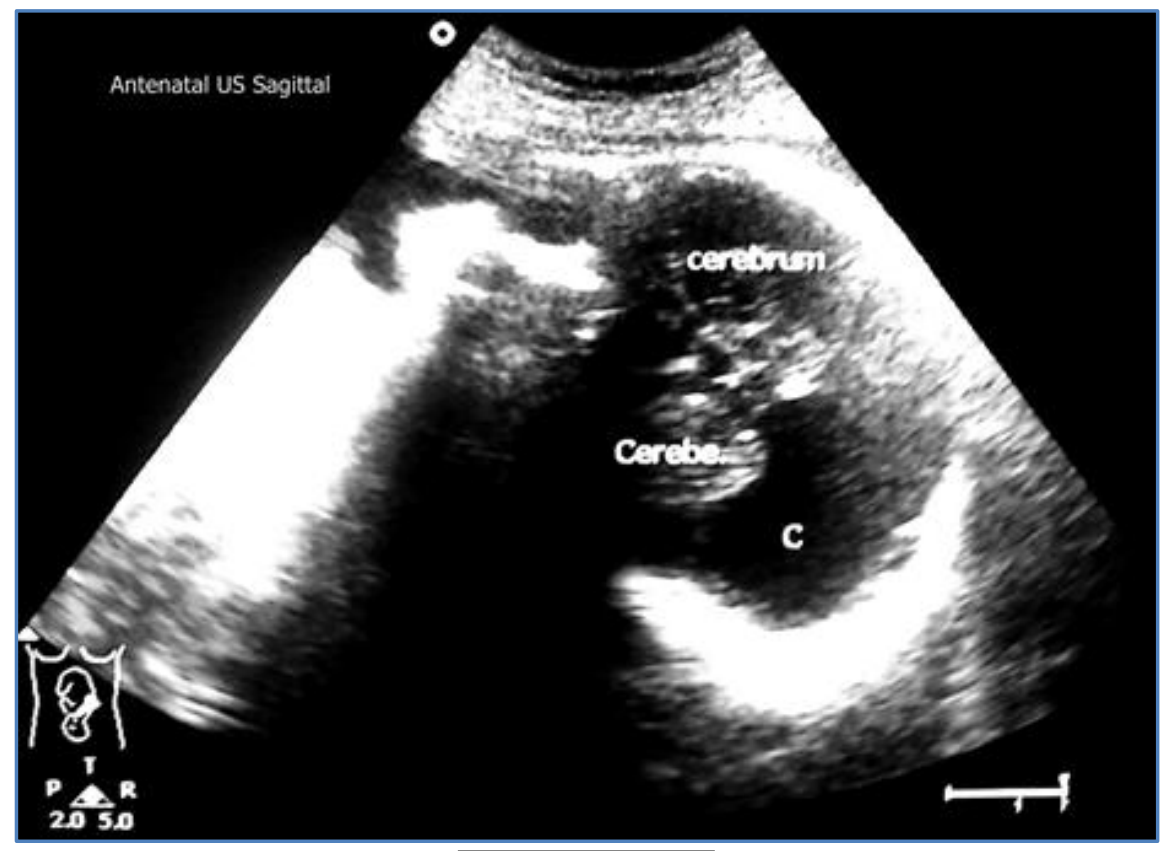

Figure 2 


\section{CASE REPORT}

Fig. 3(a, b) Non contrast axial computed tomography scan in a 3 month old infant showing marked hydrocephalus, a large cerebrospinal fluid cyst in the posterior fossa and hypoplastic cerebellar hemispheres with a "winged" appearance (C-C). Complete absence of cerebellar hemisphere with communication between cyst and fourth ventricle giving "key hole" appearancesmall arrow (c, d) Evidence of intracranial shunt in the dilated right lateral ventricle(Long arrow)

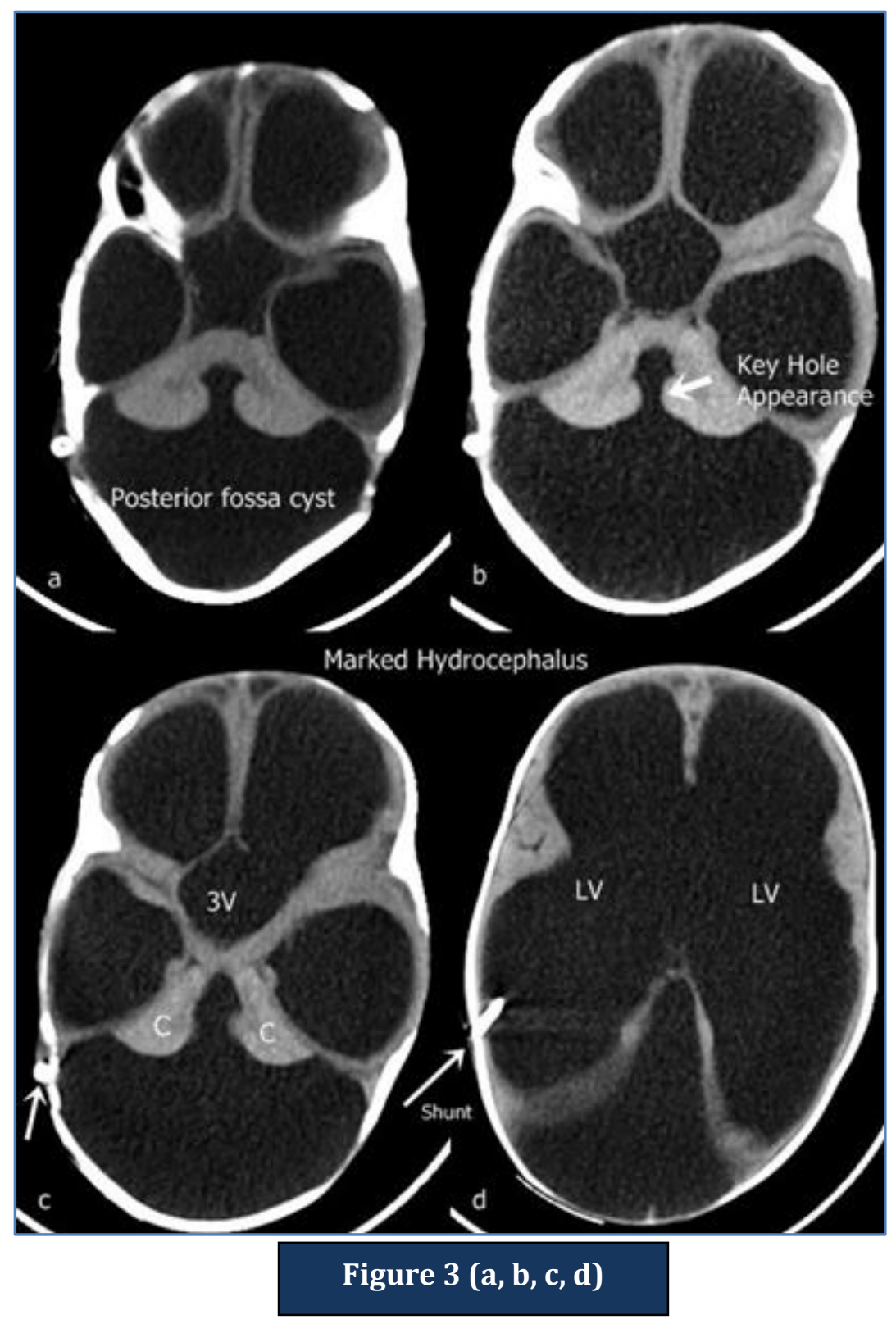




\section{CASE REPORT}

\section{AUTHORS:}

1. Arvinder Singh

2. Poonam Ohri

3. Sohan Singh

4. Manjeet Kaur

\section{PARTICULARS OF CONTRIBUTORS:}

1. Associate Professor, Department of Radiodiagnosis, G. M. C, Amritsar.

2. Assistant Professor, Department of Radiodiagnosis, G. M. C, Amritsar.

3. Professor and HOD, Department of Radiodiagnosis, G. M. C, Amritsar.

4. Associate Professor, Department of Physiology, SGRDIMSAR, Amritsar.

\section{NAME ADDRESS EMAIL ID OF THE} CORRESPONDING AUTHOR:

Dr. Arvinder Singh,

\#316-A, Moon Avenue Street No. 1,

Majitha Road,

Amritsar, Punjab,India.

E-mail: arvinderdr@rediffmail.com

Date of Submission: 23/05/2014. Date of Peer Review: 24/05/2014. Date of Acceptance: 30/05/2014. Date of Publishing: 06/06/2014. 